\title{
L'atteinte respiratoire de la leptospirose ictérohémorragique
}

\section{Respiratory Impairment with Leptospirosis Icterohaemorrhagiae (Weil's Disease)}

\author{
N. Houari $\cdot$ S. Touzani $\cdot$ N. Kanjaa
}

Reçu le 29 décembre 2017; accepté le 5 février 2018

(C) SFMU et Lavoisier SAS 2018

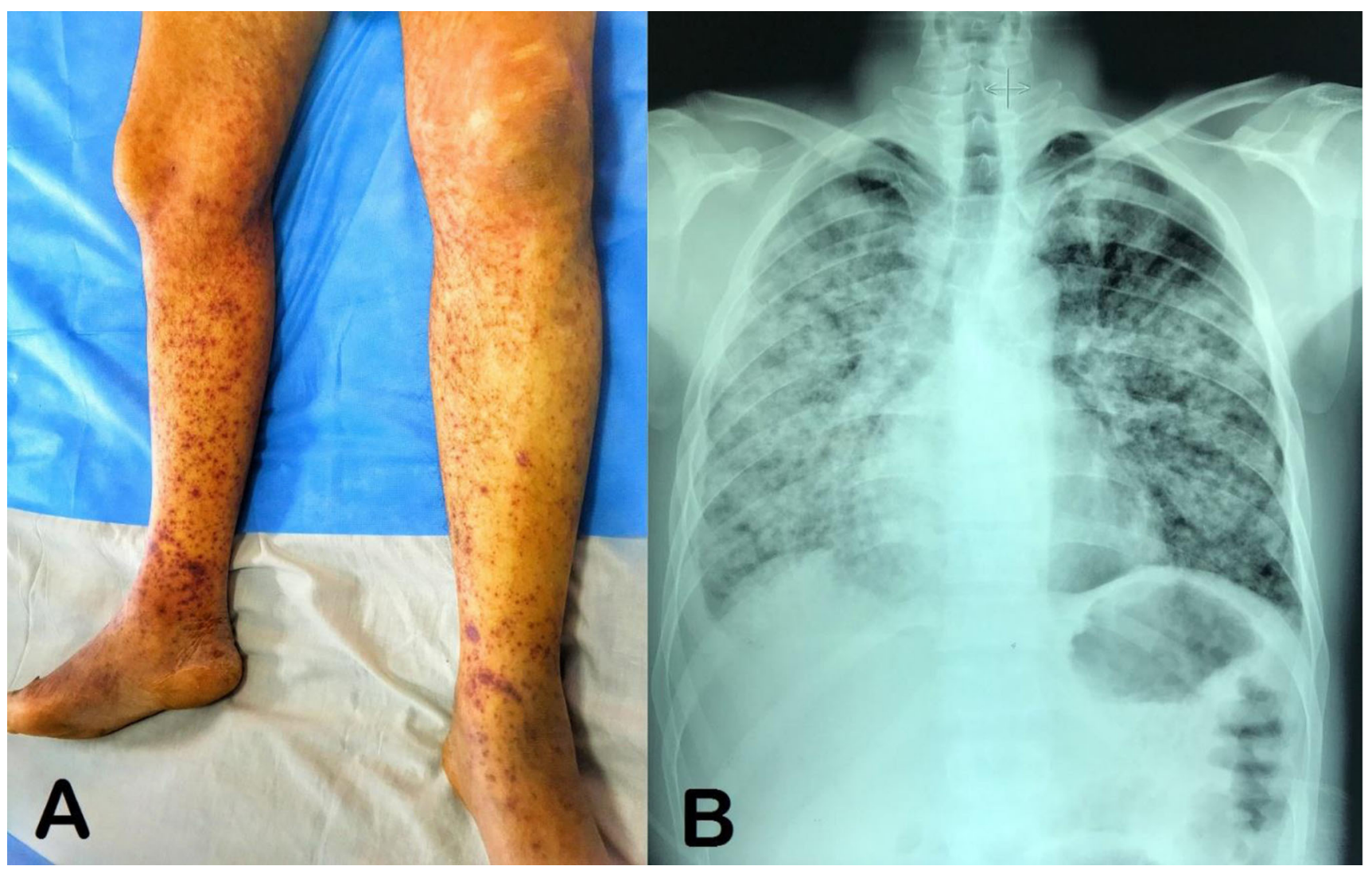

Fig. 1 A. Purpura sur fond d'ictère aux membres inférieurs. B. Radiographie thoracique : opacités alvéolaires diffuses

N. Houari $(\bowtie)$

Centre hospitalier universitaire Hassan-II de Fès, Maroc e-mail : nawfal.houari@gmail.com

\section{S. Touzani $\cdot$ N. Kanjaa}

Service de réanimation polyvalente A4,

CHU Hassan-II, Fès, Maroc

N. Houari $\cdot$ S. Touzani $\cdot$ N. Kanjaa

Faculté de Médecine et de Pharmacie de Fès,

Université Sidi Mohammed Ben Abdellah, Fès, Maroc
Un patient de 30 ans, sans antécédents notables, s'est présenté aux urgences pour des hémoptysies associées à un ictère évoluant depuis cinq jours dans les suites d'un syndrome pseudogrippal. Le patient était dyspnéique avec une $\mathrm{SpO}_{2}$ de $89 \%$ à l'air ambiant, tachycarde à 120 battements/ minute et fébrile à $38,9{ }^{\circ} \mathrm{C}$. La tension artérielle était de 130/80 mmHg. L'examen clinique retrouvait des râles crépitants bilatéraux, une sensibilité abdominale diffuse et un ictère cutanéomuqueux purpurique généralisé (Fig. 1A). La radiographie thoracique montrait des opacités alvéolaires diffuses (Fig. 1B). Le patient avait une hémoglobine à $10 \mathrm{~g} / \mathrm{dl}$, une thrombopénie à $51 \mathrm{G} / 1$, une insuffisance rénale 
à diurèse conservée avec une urée à $39 \mathrm{mmol} / \mathrm{l}$ et une créatinine à $336 \mu \mathrm{mol} / \mathrm{l}$. Les transaminases étaient à dix fois la normale. On notait une cholestase hépatique avec une bili-

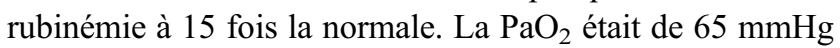
sous six litres d' $\mathrm{O}_{2}$. La troponine était négative. Les sérologies des hépatites virales $\mathrm{A}, \mathrm{B}$ et $\mathrm{C}$ étaient négatives. L'échographie hépatobiliaire et rénale ne décelait pas d'anomalie. L'atteinte hépatorénale combinée à la notion de bain maure récent faisait ainsi suspecter une leptospirose ictérohémorragique avec hémorragie alvéolaire. Le patient a bénéficié d'une antibiothérapie à base de ceftriaxone $2 \mathrm{~g} / \mathrm{j}$ et de séances de ventilation non invasive en alternance avec une oxygénothérapie au masque. La réhydratation intraveineuse était prudente pour optimiser la perfusion rénale sans aggraver la surcharge pulmonaire. L'évolution s'est faite vers une lente amélioration clinique, biologique et radiologique à j7. La sérologie de Martin et Petit était revenue positive, confirmant ainsi le diagnostic.

La leptospirose ictérohémorragique ou maladie de Weil est une anthropozoonose bactérienne ubiquitaire due à des spirochètes du genre Leptospira. Elle se présente classiquement comme une hépatonéphrite associée à une thrombopénie. L'atteinte pulmonaire est associée à une forte létalité [1]. La présentation est variable : douleur thoracique, toux, dyspnée, signes radiologiques aspécifiques, hémoptysies révélatrices d'hémorragie intra-alvéolaire ou d'œdème pulmonaire lésionnel. Le diagnostic sérologique tend progressivement à être remplacé par la réaction de polymérisation en chaîne (PCR), du fait de la haute sensibilité et spécificité de cette dernière, et de son délai de réponse rapide. Sa réalisation se limite aux sept premiers jours après le début des symptômes [2]. La prise en charge repose sur une antibiothérapie précoce (amoxicilline, céphalosporines ou doxycycline), une rééquilibration hydroélectrolytique adaptée et une éventuelle épuration extrarénale. L'oxygénation extracorporelle est indiquée en cas d'hypoxémie réfractaire. L'immunomodulation dans la phase immunitaire de la maladie de Weil est controversée.

\section{Références}

1. Dupont H, Dupont-Perdrizet D, Perie JL, et al (1997) Leptospirosis: prognostic factors associated with mortality. Clin Infect Dis 25:720-4

2. Abgueguen P (2014) Leptospirose. EMC - Traité de médecine Akos 9:1-11 